\title{
Weak Essential Submodules
}

\section{Mona A. Alsmed*}

Date of acceptance 19/1/2009

\begin{abstract}
:
A non-zero submodule $\mathrm{N}$ of $\mathrm{M}$ is called essential if $\mathrm{N} \cap \mathrm{L} \neq 0$ for each nonzero submodule $\mathrm{L}$ of $\mathrm{M}$. And a non-zero submodule $\mathrm{K}$ of $\mathrm{M}$ is called semi-essential if $\mathrm{K} \cap \mathrm{P} \neq 0$ for each non-zero prime submodule $\mathrm{P}$ of $\mathrm{M}$. In this paper we investigate a class of submodules that lies between essential submodules and semi-essential submodules, we call these class of submodules weak essential submodules.
\end{abstract}

Keywords: Semi-prime submodules, Essential submodules, Uniform modules.

\section{Introduction}

Let $\mathrm{R}$ be a commutative ring with identity 1 , and let $\mathrm{M}$ be a unitary (left) R-module.In this work we assume that every submodule of $\mathrm{M}$ contained in a semi-prime submodule of $\mathrm{M}$. A non- non-zero submodule $\mathrm{N}$ of $M$ is called essential if $N \cap L \neq(0)$ for every non-zero submodule $\mathrm{L}$ of $\mathrm{M}$ [1], and a proper submodule $\mathrm{P}$ of $\mathrm{M}$ is called prime if for each $m \in M$ and $r \in R$ whenever $r m \in M$, then either $m \in M$ or $\mathrm{r} \in[\mathrm{P}: \mathrm{M}]$ [2]. A non-zero submodule $\mathrm{K}$ of $\mathrm{M}$ is called semi-essential if $\mathrm{K} \cap \mathrm{P} \neq(0)$ for each non-zero prime submodule $\mathrm{P}$ of $\mathrm{M}$ [3].In this paper we investigate a class of submodules that lies between essential submodules and semi-essential submodules, we call this class of submodules, weak essential submodules.

\section{Notations And Basic Results:}

Recall that a submodule $\mathrm{S}$ of an $\mathrm{R}$-module $\mathrm{M}$ is called semi-prime if for each $\quad \mathrm{r} \in \mathrm{R} \quad$ and $\quad \mathrm{m} \in \mathrm{M}$ with $r^{k} x \in N, k \in Z_{+}$then $\quad \mathrm{rx} \in \mathrm{N}$ [4].Equivalently, if $r^{2} x \in N$ then $r x \in N$ [5]. In this section we study some properties of weak essential submodules.
(1.1) Definition: Let $M$ be an Rmodule. A non-zero $\mathrm{W}$ of $\mathrm{M}$ is called weak essential if $\mathrm{W} \cap \mathrm{S} \neq(0)$ for each non-zero semi-prime submodule $\mathrm{S}$ of M.

It is clear that every essential submodule is weak essential and the converse is not true in general for example: In the $Z$-module $Z_{36}$, the submodule $\overline{(9)}$ of $Z_{36}$ is weak essential but not essential, in fact $\overline{(9)} \cap \overline{(2)} \neq(0)$, $\overline{(9)} \cap \overline{(3)} \neq(0) \quad$ and $\quad \overline{(9)} \cap \overline{(6)} \neq(0)$ where $\overline{(2)}, \overline{(3)}$ and $\overline{(6)}$ are the only non-zero semi-prime submodules of Z36. But $\overline{(9)} \cap \overline{(12)}=\quad(0)$, therefore $\overline{(9)}$ is not essential submodule of $Z_{36}$. On the other hand every weak essential submodule is semi-essential, but the converse is not true as in the following example: In the Z-module $\mathrm{M}=\mathrm{Z} \oplus \mathrm{Z}$, the only prime submodule are of the form $\mathrm{Z} \oplus \mathrm{PZ}$ and $\mathrm{PZ} \oplus \mathrm{Z}$ where $\mathrm{P}$ is the prime number. The submodule $\mathrm{N}=(0) \oplus \mathrm{Z}$ of $\mathrm{M}$ is semiessential but not weak essential, since $\mathrm{N} \cap 2 \mathrm{Z} \oplus(0)=(0)$ where $2 \mathrm{Z} \oplus(0)$ is semi-prime submodule of $\mathrm{M}$ not prime submodule.

The following proposition is another characterization of weak

* Department of Mathematics. College Of Science For Women Baghdad University

E-mail: dr.muna_1965 ayahoo.com 
essential submodules. Compare with[1].

(1.2) Proposition:Let $M$ be an Rmodule. A non-zero submodule $\mathrm{W}$ of $M$ is weak essential if and only if for each non-zero semi-prime submodule $S$ of $M$ there exists $x \in S$ and $r \in R$, such that $(0) \neq \mathrm{rx} \in \mathrm{W}$.

Proof: Suppose that for each non-zero semi-prime submodule $\mathrm{S}$ of $\mathrm{M}$, there exists $x \in S$ and $r \in R$ such that $(0) \neq r x$ $\in \mathrm{W}$. Not that $r \mathrm{x} \in \mathrm{S}$, therefore $(0) \neq$ $r x \in W \cap S$. Thus $W \cap S \neq(0)$, that is $W$ is a weak essential. Conversely, suppose that $\mathrm{W}$ is a weak essential submodule of $M$. Then $W \cap S \neq(0)$ for each semiprime submodule $\mathrm{S}$ of $\mathrm{M}$, thus there exists $(0) \neq \mathrm{x} \in \mathrm{W} \cap \mathrm{S}$. This implies that $\mathrm{x} \in \mathrm{W}$ and hence $(0) \neq 1 . \mathrm{x} \in \mathrm{W}$.

A submodule $\mathrm{N}$ is called irreducible if for each two submodules $\mathrm{L}_{1}$ and $\mathrm{L}_{2}$ of $\mathrm{M}$ such that $\mathrm{L}_{1} \cap \mathrm{L}_{2}=\mathrm{N}$, then either $\mathrm{L}_{1}=\mathrm{N}$ or $\mathrm{L}_{2}=\mathrm{N}$ [4]. We can show that if every semi-prime submodule of $\mathrm{M}$ is irreducible then a semi-essential submodule is weak essential as in the following proposition. Before that we need the following lemma which the proof can be seen in [5]

(1.3) Lemma: Let $S$ be an irreducible submodule of $\mathrm{M}$. Then $\mathrm{S}$ is semi-prime if and only if $\mathrm{S}$ is prime submodule.

(1.4) Proposition: Let $M$ be an Rmodule such that every semi-prime submodule of $\mathrm{M}$ is irreducible. If a submodule $\mathrm{W}$ of $\mathrm{M}$ is semi-essential then $\mathrm{W}$ is a weak essential submodule of $\mathrm{M}$.

Proof: Let $\mathrm{S}$ be a non-zero semi-prime submodule of $\mathrm{M}$ with $\mathrm{W} \cap \mathrm{S}=(0)$. Since $\mathrm{S}$ is irreducible submodule then by (1.3), $\mathrm{S}$ is prime submodule. But $\mathrm{W}$ is semi-essential submodule of $M$, therefore $\mathrm{S}=(0)$
(1.5) Remarks:

1. If $\mathrm{W}$ is a weak essential submodule and $\mathrm{N}$ is a submodule of $\mathrm{W}$ then $\mathrm{N}$ need not be weak essential. For example: consider the $\mathrm{Z}$-module $\mathrm{Z}_{36}$, the submodule $\overline{(2)}$ of $Z_{36}$ is weak essential but the submodule $\overline{(18)}$ of $\overline{(2)}$ is not weak essential since $\overline{(18)} \cap \overline{(12)}=\overline{(0)}$ where $\overline{(12)}$ is a semiprime submodule of $\overline{(2)}$.

2. Let $\mathrm{M}$ be an $\mathrm{R}$-module and let $\mathrm{W}_{1}$ and $\mathrm{W}_{2}$ be submodules of $\mathrm{M}$ such that $\mathrm{W}_{1} \subseteq \mathrm{W}_{2}$. If $\mathrm{W}_{1}$ is a weak essential submodule of $M$ then $W_{2}$ is weak essential submodule of $\mathrm{M}$.

3. Let $\mathrm{M}$ be an R-module, and let $\mathrm{W}_{1}$ and $\mathrm{W}_{2}$ be submodules of $\mathrm{M}$, if $\mathrm{W}_{1} \cap \mathrm{W}_{2}$ is a weak essential submodule of $\mathrm{M}$, then both of $\mathrm{W}_{1}$ and $\mathrm{W}_{2}$ are weak essential submodules of M.

Proof:

(2). Assume that $\mathrm{W}_{2} \cap \mathrm{S}=(0)$, for some semi-prime submodule $\mathrm{S}$ of $\mathrm{M}$, then $\mathrm{W}_{1} \cap \mathrm{S}=(0)$. But $\mathrm{W}_{1}$ is a weak essential submodule of $\mathrm{M}$, therefore $\mathrm{S}=(0)$ and hence we are done.

(3). Follows immediately from (2).

The converse of (3) is not true in general for example, in the Zmodule $Z_{36}$ the only non-zero semiprime submodules are only $\overline{(2)}, \overline{(3)}$ and $\overline{(6)}$. Both of $\overline{(12)}$ and $\overline{(18)}$ are weak essential submodules, but the intersection $\overline{(12)} \cap \overline{(18)}=\overline{(0)}$ is not weak essential submodule of $Z_{36}$.

Under some conditions the converse of (3) will be true as in the following two propositions.

(1.6) Proposition: Let $M$ be an Rmodule and let $\mathrm{W}_{1}$ and $\mathrm{W}_{2}$ be submodules of $M$ such that $W_{1}$ is an essential submodule of $\mathrm{M}$, and $\mathrm{W}_{2}$ is 
weak essential submodule of $\mathrm{M}$. Then $\mathrm{W}_{1} \cap \mathrm{W}_{2}$ is weak essential submodule of $\mathrm{M}$.

Proof: Since $W_{2}$ is a weak essential submodule of $M$, then $W_{2} \cap S \neq(0)$ for each non-zero semi-prime submodule $\mathrm{S}$ of $\mathrm{M}$. But $\mathrm{W}_{1}$ is an essential submodule of $\mathrm{M}$, so $\mathrm{W}_{1} \cap\left(\mathrm{W}_{2} \cap \mathrm{S}\right)$ $\neq(0)$, this implies that $\left(\mathrm{W}_{1} \cap \mathrm{W}_{2}\right) \cap \mathrm{S}$ $\neq(0)$, thus we get the result.

(1.7) Proposition: Let $M$ be an Rmodule and let $W_{1}$ and $W_{2}$ be submodules of $\mathrm{M}$ such that one of them does not contained in any semiprime submodule of $\mathrm{M}$. If $\mathrm{W}_{1}$ and $\mathrm{W}_{2}$ are weak essential submodules of $\mathrm{M}$, then $\mathrm{W}_{1} \cap \mathrm{W}_{2}$ is weak essential submodule of $\mathrm{M}$.

Proof: Suppose that there exists a semi-prime submodule $\mathrm{S}$ of $\mathrm{M}$ such that $\left(\mathrm{W}_{1} \cap \mathrm{W}_{2}\right.$.) $\cap \mathrm{S}=(0)$ Then $\mathrm{W}_{1} \cap$ $\left(\mathrm{W}_{2} \cap \mathrm{S}\right)=(0)$. By assumption either $\mathrm{W}_{1}$ or $\mathrm{W}_{2}$ is not contained in $\mathrm{S}$. If $\mathrm{W}_{2} \not \subset$ $\mathrm{S}$, then $\mathrm{W}_{2} \cap \mathrm{S}$ is semi-prime submodule of $W_{2}$ [5]. But $W_{1}$ is weak essential submodule of $\mathrm{M}$, so $\mathrm{W}_{2} \cap \mathrm{S}=$ (0). Also $\mathrm{W}_{2}$ is weak essential submodule of $\mathrm{M}$, therefore $\mathrm{S}=(0)$.

\section{$\xi$ 2. Weak essential homomorphisms:}

This section is devoted to study weak essential homomorphisms, we start by the following definition.

(2.1) Definition: Let $M_{1}$ and $M_{2}$ be two R-modules. An R-homomorphism $\mathrm{f}: \mathrm{M}_{1} \rightarrow \mathrm{M}_{2}$ is called essential homomorphism if $\mathrm{f}\left(\mathrm{M}_{1}\right)$ is a weak essential submodule of $\mathrm{M}_{2}$.

(2.2) Remark: Let $M$ be an R-module and let $\mathrm{W}$ be a submodule of $\mathrm{M}$. W is weak essential submodule if and only if the inclusion homomorphism $\mathrm{i}$ :
$\mathrm{W} \rightarrow \mathrm{M}$ is weak essential homomorphism.

Compare the following proposition with [6].

(2.3) Proposition: Let $M_{1}$ and $M_{2}$ be R-modules and let $\mathrm{f}: \mathrm{M}_{1} \rightarrow \mathrm{M}_{2}$ be an R-epimorphism, then:

1. If $W_{1}$ is a weak essential submodule of $M_{1}$, then $f\left(W_{1}\right)$ is weak essential submodule of $\mathrm{M}_{2}$

2. If $\mathrm{W}_{2}$ is a weak essential submodule of $\mathrm{M}_{2}$ such that ker (f) $\subseteq \mathrm{S}_{1}$ for each semi-prime submodule $\mathrm{S}_{1}$ of $\mathrm{M}_{1}$, then $f^{-1}\left(W_{2}\right)$ is weak essential submodule of $\mathrm{M}_{1}$.

Proof:

1. Let $\mathrm{S}_{2}$ be a non-zero semi-prime submodule of $\mathrm{M}_{2}$, then $f^{-1}\left(S_{2}\right)$ is semiprime submodule of $M_{1}$ [5]. But $W_{1}$ is weak essential submodule of $\mathrm{M}_{1}$, thus $W_{1} \cap f^{-1}\left(S_{2}\right) \neq(0)$ and hence $\mathrm{f}\left(\mathrm{W}_{1}\right)$ $\cap \mathrm{S}_{2} \neq(0)$.

2. Suppose there exists a non-zero semi-prime submodule $\mathrm{S}_{1}$ of $\mathrm{M}_{1}$ such that $f^{-1}\left(W_{2}\right) \cap S_{1}=(0)$, this implies that $\mathrm{W}_{2} \cap f\left(\mathrm{~S}_{1}\right)=(0)$. But $\mathrm{S}_{1}$ is semiprime submodule with $\operatorname{ker}(\mathrm{f}) \subseteq \mathrm{S}_{1}$, so $\mathrm{f}\left(\mathrm{S}_{1}\right)$ is semi-prime submodule of $\mathrm{M}_{2}$ [5]. But $\mathrm{W}_{2}$ is weak essential submodule of $\mathrm{M}_{2}$, therefore $\mathrm{f}\left(\mathrm{S}_{1}\right)=(0)$ which implies that $\mathrm{S}_{1} \subseteq \operatorname{ker}(\mathrm{f})$ $\subseteq f^{-1}\left(W_{2}\right)$, and hence $\mathrm{S}_{1}=f^{-1}\left(W_{2}\right) \cap$ $\mathrm{S}_{1}=(0)$ that is $\mathrm{S}_{1}=(0)$.

Analogue of proposition (2.3.6) in [7] we can prove the following lemma which we need it in the next theorem.

(2.4) Lemma: Let $M_{1}$ and $M_{2}$ be Rmodules and let $\mathrm{W}_{2}$ be a semi-prime submodule of $\mathrm{M}_{2}$ such that $\operatorname{Hom}_{R}\left(M_{1}, W_{2}\right) \subset \operatorname{Hom}_{R}\left(M_{1}, M_{2}\right)$, 
then $\operatorname{Hom}_{R}\left(M_{1}, W_{2}\right)$ is semi-prime submodule of $\operatorname{Hom}_{R}\left(M_{1}, M_{2}\right)$.

Proof: Let $\quad \mathrm{r} \in \mathrm{R} \quad$ and $\mathrm{f} \in \operatorname{Hom}_{R}\left(M_{1}, M_{2}\right) \quad$ such that $\mathrm{r}^{2} \mathrm{f} \in \operatorname{Hom}_{R}\left(M_{1}, W_{2}\right)$ then for each $\mathrm{x} \in$ $\mathrm{M}_{1}, \mathrm{r}^{2} \mathrm{f}(\mathrm{x}) \in \mathrm{W}_{2}$. But $\mathrm{W}_{2}$ is semi-prime submodule of $\mathrm{M}_{2}$, so $\mathrm{rf}(\mathrm{x}) \in \mathrm{W}_{2}$, hence rf $\in \operatorname{Hom}_{R}\left(M_{1}, W_{2}\right)$.

(2.5) Theorem: Let $\mathrm{M}_{1}$ and $\mathrm{M}_{2}$ be $\mathrm{R}$ modules, and let $\operatorname{Hom}_{R}\left(M_{1}, W_{2}\right)$ be a proper submodule of $\operatorname{Hom}_{R}\left(M_{1}, M_{2}\right)$ for any submodule $\mathrm{W}_{2}$ of $\mathrm{M}_{2}$. If $\operatorname{Hom}_{R}\left(M_{1}, W_{2}\right)$ is weak essential submodule of $\operatorname{Hom}_{R}\left(M_{1}, M_{2}\right)$, then $\mathrm{W}_{2}$ is weak essential submodule of $\mathrm{M}_{2}$.

Proof: Let $\mathrm{S}_{2}$ be a non-zero semiprime submodule of $\mathrm{M}_{2}$.By (2.4), $\operatorname{Hom}_{R}\left(M_{1}, S_{2}\right)$ is semi-prime submodule of $\operatorname{Hom}_{R}\left(M_{1}, M_{2}\right)$.But $\operatorname{Hom}_{R}\left(M_{1}, W_{2}\right)$ is weak essential submodule of $\operatorname{Hom}_{R}\left(M_{1}, M_{2}\right)$ then by (1.2), there exists $0 \neq \mathrm{f} \in \operatorname{Hom}_{R}\left(M_{1}, S_{2}\right)$ and $0 \neq \mathrm{r} \in \mathrm{R}$ such that $0 \neq \mathrm{rf} \in \operatorname{Hom}_{R}\left(M_{1}, W_{2}\right)$, that is $\operatorname{rf}(m) \in \mathrm{W}_{2}$ for each $\mathrm{m} \in \mathrm{M}_{1}$. So for each non-zero semi-prime submodule $\mathrm{S}_{2}$ of $M_{2}$ we find $f(m) \in S_{2}$ for each $m \in M_{1}$ and we find $r \in R$ with $0 \neq r f(m) \in W_{2}$ i.e. $\mathrm{W}_{2}$ is essential submodule of $\mathrm{M}_{2}$.

(2.6) Corollary: Let $M$ be an Rmodule and let $\mathrm{W}$ be a submodule of $\mathrm{M}$.If $\operatorname{Hom}_{R}(M, W)$ is weak essential submodule of $\operatorname{Hom}_{R}(M, M)$, then $\mathrm{W}$ is weak essential submodule of $\mathrm{M}$.

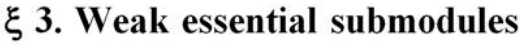 in multiplication modules}

Recall that an R-module $\mathrm{M}$ is called multiplication if for each submodule $\mathrm{N}$ of $\mathrm{M}$ there exists an ideal
I of $\mathrm{R}$ such that $\mathrm{N}=\mathrm{IM}$ [8]. ].A nonzero ideal $\mathrm{I}$ of $\mathrm{R}$ is called weak essential if $I \cap S \neq(0)$ for each non-zero semi-prime ideal $\mathrm{S}$ of $\mathrm{R}$.

(3.1) Proposition: Let $M$ be a finitely generated faithful multiplication module. And let $\mathrm{W}$ be a submodule of $\mathrm{M}$ such that $\mathrm{W}=\mathrm{IM}$ for some ideal I of $\mathrm{R}$. If $\mathrm{W}$ is a weak essential submodule of $M$ then I is weak essential ideal of $\mathrm{R}$.

Proof: Suppose that $I \cap S=(0)$ for some non-zero semi-prime ideal $\mathrm{S}$ of R. Since $M$ is a faithful multiplication module, then $(0)=(I \cap S) M=I M \cap S M$. Also since $\mathrm{S}$ is semi-prime submodule, and $M$ is finitely generated multiplication module so by [5], SM is semi-prime submodule of $\mathrm{M}$. On the other hand $\mathrm{W}=\mathrm{IM}$ is weak essential submodule of $\mathrm{M}$, therefore $\mathrm{SM}=(0)$. But $\mathrm{M}$ is faithful module then $\mathrm{S}=(0)$.

Under some conditions the converse of (3.2) is true as in the following two propositions.

(3.2) Proposition: Let $M$ be a faithful multiplication module and let $\mathrm{W}$ be submodule of $\mathrm{M}$ such that $\mathrm{W}=\mathrm{IM}$. Suppose that every non-zero proper semi-prime submodule of $\mathrm{M}$ is irreducible. If I is weak essential ideal of $\mathrm{R}$ then $\mathrm{W}$ is a weak essential submodule of $\mathrm{M}$.

Proof: Suppose that $\mathrm{W} \cap \mathrm{S}=(0)$ for some non-zero proper semi-prime submodule $\mathrm{S}$ of $\mathrm{M}$. By assumption $\mathrm{S}$ is an irreducible submodule of $\mathrm{M}$, so by (1.3), $\mathrm{S}$ is prime submodule. But $\mathrm{S}$ is a proper submodule of the multiplication module $\mathrm{M}$, this implies that there exists a prime ideal $P$ of $R$ such that $S=P M$ [8]. Now $(0)=\mathrm{W} \cap \mathrm{S}=\mathrm{IM} \cap \mathrm{PM}=(\mathrm{I} \cap \mathrm{P})$ M. But $\mathrm{M}$ is faithful multiplication module, therefore $\mathrm{I} \cap \mathrm{P}=(0)$. Since every prime submodule is semi-prime 
submodule, and by assumption we get $\mathrm{P}=(0)$. But $\mathrm{S}=\mathrm{PM}$ therefore $\mathrm{S}=(0)$.

(3.3) Proposition: Let $M$ be a faithful multiplication module and let $\mathrm{W}$ be submodule of $\mathrm{M}$ such that $\mathrm{W}=\mathrm{IM}$. Suppose that every non-zero proper semi-prime submodule of $M$ is primary. If I is weak essential ideal of $\mathrm{R}$ then $\mathrm{W}$ is weak essential submodule of $\mathrm{M}$.

Proof: Suppose that $\mathrm{W} \cap \mathrm{S}=(0)$ for some non-zero proper semi-prime submodule $\mathrm{S}$ of $\mathrm{M}$. By assumption $\mathrm{S}$ is a primary submodule of $\mathrm{M}$. Since $\mathrm{M}$ is multiplication module then [S: M] is semi-prime submodule of $\mathrm{M}$ [5]. But $\mathrm{S}$ is primary submodule of $\mathrm{M}$, therefore $\mathrm{S}$ is a prime submodule [6], this implies there exists a prime ideal $\mathrm{P}$ of $\mathrm{R}$ such that $\mathrm{S}=\mathrm{PM}$ [8]. Now $(0)=$ $\mathrm{W} \cap \mathrm{S}=\mathrm{IM} \cap \mathrm{PM}=(\mathrm{I} \cap \mathrm{P}) \mathrm{M}$. But $\mathrm{M}$ is faithful multiplication

module, therefore $I \cap P=(0)$. Since every prime submodule is semi-prime submodule, and by assumption we get $\mathrm{P}=(0)$. But $\mathrm{S}=\mathrm{PM}$ therefore $\mathrm{S}=(0)$.

(3.4) Proposition: Let $M$ be a finitely generated faithful multiplication module and let $\mathrm{W}$ be a submodule of M. If $\mathrm{W}$ is weak essential submodule of $M$ then $[\mathrm{W}:(\mathrm{m})]$ is weak essential ideal of $\mathrm{R}$ for each $\mathrm{m} \in \mathrm{M}$. The converse is true if every non-zero proper semi-prime submodule of $M$ is irreducible.

Proof: Assume that $\mathrm{W}$ is weak essential submodule of M. By (3.2), [W: M] is weak essential ideal of $\mathrm{R}$. But for each $\mathrm{m} \in \mathrm{M},[\mathrm{W}: \mathrm{M}] \subseteq[\mathrm{W}:($ $\mathrm{m})$ ]. Since $M$ is faithful multiplication, thus $[\mathrm{N}: \mathrm{M}] \mathrm{M} \subseteq[\mathrm{W}:(\mathrm{m})] \mathrm{M}[8]$. This implies that $[\mathrm{W}:(\mathrm{m})] \mathrm{M}$ is a weak essential submodule of $M$ (1.5) (2). Hence $[\mathrm{W}:(\mathrm{m})]$ is weak essential ideal of $\mathrm{R}$ (3.2). Conversely, assume that $[\mathrm{W}:(\mathrm{m})]$ is a weak essential ideal of $\mathrm{R}$ for each $\mathrm{m} \in \mathrm{M}$, and let $\mathrm{S}$ be a non-zero proper semi-prime submodule of $M$. Since $M$ is a multiplication module and $\mathrm{S}$ is irreducible submodule, then by (1.3), $\mathrm{S}$ is prime submodule, so there exists a prime ideal $\mathrm{P}$ of $\mathrm{R}$ such that $\mathrm{S}=\mathrm{PM}$ [8]. It is clear that $\mathrm{P}$ is semi-prime ideal of $\mathrm{R}$, but $[\mathrm{W}:(\mathrm{m})]$ is weak essential ideal of $\mathrm{R}$, therefore $[\mathrm{W}:(\mathrm{m})] \cap \mathrm{P} \neq(0)$. Since $M$ is a faithful multiplication module, then [W: $(\mathrm{m})] \mathrm{M} \cap \mathrm{PM} \neq(0)$. Thus $\mathrm{W} \cap \mathrm{S} \neq(0)$ that is $\mathrm{W}$ is a weak essential submodule of $\mathrm{M}$.

By the same way we can prove the following.

(3.5) Proposition: Let $M$ be a finitely generated faithful multiplication module and let $\mathrm{W}$ be a submodule of M. If $\mathrm{W}$ is weak essential submodule of $M$ then $[W:(m)$ ] is weak essential ideal of $R$ for each $m \in M$. The converse is true if every non-zero proper semi-prime submodule of $M$ is primary.

From the last four propositions we have the following two theorems.

(3.6) Theorem: Let $M$ be a finitely generated faithful multiplication module, and let $\mathrm{W}$ be a submodule of $\mathrm{M}$ such that $\mathrm{W}=\mathrm{IM}$ for some ideal I of $\mathrm{R}$. If each non-zero proper semi-prime submodule of $\mathrm{M}$ is irreducible, then the following statements are equivalent.

1. W is a weak essential submodule of M.

2. I is a weak essential ideal of $R$.

3. $[\mathrm{W}:(\mathrm{m})]$ is a weak essential ideal of $\mathrm{R}$ for each $\mathrm{m} \in \mathrm{M}$.

Proof: (1) $\Rightarrow(2)$ : By (3.2).

(2) $\Rightarrow(3)$ : Assume that I is an essential ideal of $R$. Since $M$ is finitely generated faithful module, then by [5], $\mathrm{I}=[\mathrm{IM}: \mathrm{M}]$. But $[\mathrm{IM}: \mathrm{M}] \subseteq[\mathrm{IM}:(\mathrm{m})]$ for each $m \in M$, and [IM:M] is a weak 
essential ideal of $\mathrm{R}$, also we consider [IM:M] as an R-module, then by $(1.4)(2),[M:(m)]$ is a weak essential submodule of $\mathrm{R}$, hence we get the result.

$$
\text { (3) } \Rightarrow \text { (1): By (3.5). }
$$

(3.7) Theorem: Let $M$ be a finitely generated faithful multiplication module, and let $\mathrm{W}$ be a submodule of $M$ such that $\mathrm{W}=\mathrm{IM}$ for some ideal $\mathrm{I}$ of $\mathrm{R}$. If each non-zero proper semi-prime submodule of $\mathrm{M}$ is primary then the following statements are equivalent.

1. W is a weak essential submodule of M.

2. I is a weak essential ideal of $R$.

3. $[\mathrm{W}:(\mathrm{m})]$ is a weak essential ideal of $\mathrm{R}$ for each $\mathrm{m} \in \mathrm{M}$.

Proof: By the same way of (3.6), only in the direction $\mathbf{( 3 )} \Rightarrow(\mathbf{1})$ we depend on (3.5)

\section{$\xi$ 4. Weak uniform modules}

Recall that a non-zero Rmodule $\mathrm{M}$ is called uniform if every non-zero submodule of $\mathrm{M}$ is an essential submodule [6]. Abdullah, N.K. gave in her thesis [3] a generalization of uniform modules, she name it semi-uniform module that is a module $\mathrm{M}$ in which every non-zero submodule is semi-essential. In this section we introduce another generalization of uniform modules in fact this class of modules lies between uniform modules and semi-uniform modules. We call it weak uniform modules. We start by the following definition.

(4.1) Definition: A non-zero module $\mathrm{M}$ is called weak uniform, if each nonzero submodule of $\mathrm{M}$ is weak essential. And a ring $\mathrm{R}$ is called uniform ring if it is uniform module as an R-module.

\section{(4.2) Remarks:}

1. It is clear that each uniform module is weak uniform module. However, the converse is not true in general, for example: The Z-module $Z_{36}$ is a weak uniform. In fact the only non-zero semi-prime submodule of $Z_{36}$ $\operatorname{are} \overline{(2)}, \overline{(3)} \& \overline{(6)}$ and all of them have non-zero intersections with each non trivial submodule of $Z_{36}$ which they are $\overline{(2)}, \overline{(3)}, \quad \overline{(4)}, \quad \overline{(6)}$ and $\overline{(9)}, \overline{(12)}$ and $\overline{(18)}$. Therefore all submodules of $Z_{36}$ are weak essential. On the other hand $\overline{(18)} \cap \overline{(12)}=\overline{(0)}$, this mean $\overline{(18)}$ is not essential submodule of $Z_{36}$. Thus $\mathrm{Z}_{36}$ is not uniform module.

2. Also it can be easy shown that each weak uniform module is semi-uniform. The converse is not true in general. For example the submodule $\overline{(2)}$ of $Z_{36}$ is semi-uniform since the only non-zero semi-prime submodules of (2) are $\overline{(4)} \& \overline{(6)}$ and the last submodules have non-zero intersections with each non trivial submodule of $\overline{(2)}$. On the other hand the submodule $\overline{(2)}$ is not weak uniform since it is contain a submodule $\overline{(18)}$ which is not weak essential because $\overline{(18)} \cap \overline{(12)}=\quad(0)$ where $\overline{(12)}$ is semi-prime submodule of $\overline{(2)}$.

It is shown in [3] that the uniform property is hereditary. Now we show by example that the weak uniform property is not hereditary. The $\mathrm{Z}$-module $\mathrm{Z}_{36}$ is weak uniform module (4.2) (1). But $\overline{(3)}$ is not weak uniform submodule of $Z_{36}$ since $\overline{(12)}$ is not weak essential submodule of $\overline{(3)}$, the only non-zero semi-prime submodule of $\overline{(3)}$ are $\overline{(6)}, \quad \overline{(9)} \& \overline{(18)} \quad$ while $\overline{(12)} \cap \overline{(18)}=\overline{(0)}$. 
Compare the following proposition with [3].

(4.3) Theorem: Let $M$ be a finitely generated faithful and multiplication $\mathrm{R}$-module. Then $\mathrm{M}$ is a weak uniform module if and only if $\mathrm{R}$ is weak uniform ring.

Proof: Assume that $M$ is a weak uniform module, and let I be a nonzero ideal of $R$ such $I \cap S=(0)$ for each non-zero semi-prime ideal $\mathrm{S}$ of $\mathrm{R}$. Since $\mathrm{M}$ is a multiplication module, so $\mathrm{IM} \cap \mathrm{SM}=(0)$ [9]. On the other hand because of $\mathrm{M}$ is multiplication and $\mathrm{S}$ is a semi-prime ideal of $\mathrm{R}$ therefore $\mathrm{SM}$ is semi-prime submodule of M [5]. But $\mathrm{M}$ is weak uniform module and IM is a submodule of $\mathrm{M}$, so $\mathrm{SM}=(0)$. Since $\mathrm{M}$ is faithful module, then $S=(0)$ and hence $I$ is weak essential ideal of $R$. Conversely, let $\mathrm{R}$ be a weak uniform ring, and let $\mathrm{W}$ be a non-zero submodule of $\mathrm{M}$ and $\mathrm{S}$ be a non-zero semi-prime submodule of $\mathrm{M}$ such that $\mathrm{W} \cap \mathrm{S}=(0)$. Thus $[\mathrm{W}: \mathrm{M}] \cap[\mathrm{S}: \mathrm{M}]=$ (0). But [S: M] is semi-prime ideal of $\mathrm{R}$ [5], and $\mathrm{R}$ is a weak uniform ring, so $[S: M]=(0)$ which implies that $S=$ (0). That is $\mathrm{W}$ is weak essential submodule of $\mathrm{M}$.

(4.4) Theorem: Let $M$ be an R-module and let $\mathrm{N}$ be an essential submodule of $\mathrm{M}$ such that $\mathrm{N}$ does not contained in any semi-prime submodule of $\mathrm{M}$. If $\mathrm{N}$ is a weak uniform submodule then $M$ is weak uniform module.

Proof: Let $\mathrm{K}$ be any submodule of $\mathrm{M}$ with $\mathrm{K} \cap \mathrm{S}=(0)$ for each non-zero semi-prime submodule $\mathrm{S}$ of $\mathrm{M}$. So $\mathrm{N} \cap$ $(\mathrm{K} \cap \mathrm{S})=(0)$, and then $(\mathrm{N} \cap \mathrm{K}) \cap(\mathrm{N} \cap$ $\mathrm{S})=(0)$. By assumption, $\mathrm{N} \not \subset \mathrm{S}$ then $\mathrm{N} \cap$ $\mathrm{S}$ is a semi-prime submodule of $\mathrm{N}$ [6]. On the other hand $\mathrm{N} \cap \mathrm{K}$ is a submodule of $\mathrm{N}$, and $\mathrm{N}$ is a weak uniform, therefore $(N \cap S)=(0)$. Since $\mathrm{N}$ is essential submodule of $\mathrm{M}$, then $\mathrm{S}=$ (0).

(4.5) Corollary: Let $M$ be an Rmodule such that $\mathrm{M}$ does not contained in any semi-prime submodule of $\mathrm{E}$ (M). If $\mathrm{M}$ is a weak uniform module then $\mathrm{E}(\mathrm{M})$ is weak uniform module where $\mathrm{E}(\mathrm{M})$ is the injective hull of $\mathrm{M}$.

Proof: By assumption $\mathrm{M}$ is an essential submodule of $\mathrm{E}(\mathrm{M})$, and by (4.4) we get the result.

\section{References}

1. Kasch, F.1982, Modules and Rings, Academic press, London.

2. Lu, C.P.1984, Prime submodules of modules, Comment.Math.Univ.St. Paul. 33:61-69.

3. Abdullah N.K. 2005, Semiessential submodules and semiuniform modules, M. Sc. Thesis, Univ. of Tkrit.

4. Dauns, J. and McDonald, B.R.1980, Prime modules and onesided ideals in ring theory and algebra III , Proceedings of the third Oklahoma conference:301344.

5. Athab, E.A.1996, Prime and semiprime submodules, M. Sc. Thesis, Univ. of Baghdad.

6. Goodearl, K.R.1976, Ring theory, Marcel Dekker, New York.

7. Abdul-Razak, H.M.1999, Quasiprime modules and quasi-prime submodules,M.Sc. Thesis, Univ. of Baghdad.

8. Elbast, Z. A. and Smith, P. F. 1988, Multiplication modules, Comm. Algebra Vol. 16:755-774.

9. Ahmed, A. A. 1992, on submodules of multiplication modules, M. Sc. Thesis, Univ. of Baghdad.

$$
\text { المقاسات الجزئية الجوهرية الضعيفة }
$$




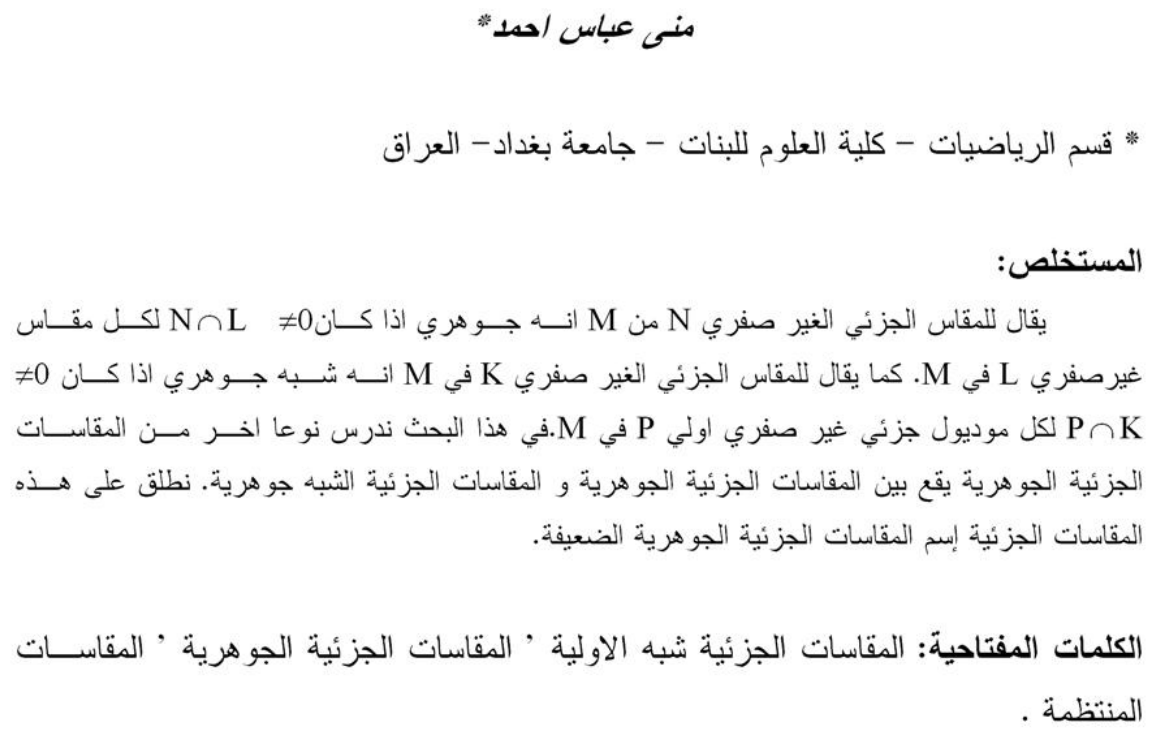

